\title{
EL NIÑO Queer O CRECER OBLICUAMENTE EN EL SIGLO VEINTE, POR KATHRYN BOND STOCKTON
}

\author{
Ana M. Cortés*
}

\section{Resumen}

The Queer Child, por Kathryn Bond se sitúa en un lugar incómodo entre las teorías de género y las de la infancia. Ha sido criticado por no ajustarse a las formas tradicionales de acercarse a los estudios de la infancia, y mal comprendido como una defensa de que todos los niños son homosexuales. La aproximación de Bond Stockton no es histórica ni psicológica, sino que se sitúa en el ámbito más amplio y difuso de la teoría-crítica y los estudios culturales. Desde allí, pretende descubrir los imaginarios sociales en torno a la niñez en objetos tales como el cine y la literatura. Este estudio, a través del campo de la cultura, revela que no todo niño es homosexual, sino más bien, todo niño es queer, puesto que se encuentra en un tránsito ambiguo hacia la adultez, fuera y dentro del mundo de los adultos, y es constantemente amenazado por las restricciones sociales que intentan protegerlo.

Palabras clave: infancia, queer, estudios culturales, crecer oblicuamente.

\section{The Queer CHILD or GROWING SIDEWAYS IN THE TWENTIETH CENTURY, BY KATHRYN BOND STOCKTON}

\begin{abstract}
This work by Kathryn Bond is situated in an uncomfortable place between gender theories and childhood studies. It has been criticized for not adjusting to traditional approaches to the study of childhood, and miss-understood as defense of the thesis that every child is a homosexual. Bond Stockton's approach is not historical nor psychological, it lies on the more diffuse and broad ambit of critical theories and cultural studies. From that place, she attempts to unveil the social imaginaries about childhood in cultural objects such as film and literature. This study throughout the field of culture reveals not that every child is homosexual, but rather that every child is queer, for its nature is that of an ambiguous passage to adulthood, that places the child not inside nor outside the adult world, and is constantly threatened by the constraints that are meant to protect him.
\end{abstract}

Keywords: childhood, queer, cultural studies, growing sideways.

* Licenciada en Letras Hispánicas de la Pontificia Universidad Católica de Chile, Santiago, Chile. Correo electrónico: amcorte1@uc.cl. 


\title{
1. Encontrar al niño queer en el espacio teórico
}

\author{
"What a child 'is' is a darkening question." \\ ["Qué 'es' un niño es una pregunta ensombrecedora”] \\ - K. Bond Stockton.
}

Esta publicación de Kathryn Bond Stockton -aunque no es recienteha sido escasamente reseñada en español $\mathrm{y}$, a veces, torpemente leída en su propia lengua. Algunas de estas lecturas, como la de Frank A. Salamone, publicada el 2012 por la revista Childhood, reduce la tesis de la investigación a una defensa de que todos los niños son homosexuales; otras, como la de Don Romesburg, publicada en H-Childhood (2010), critican que el de Bond Stockton no sea un trabajo histórico más acabado. Aunque no viene al caso desentrañar aquí las raíces de la recepción más o menos afortunada de este libro, parece claro que The Queer Child se sitúa en un lugar incómodo al interior del ya complejo campo de los estudios de la infancia. La propuesta de Bond Stockton, a diferencia de los estudios más reconocidos como el de Philippe Ariès o Lloyd deMause, no proviene ni de la historia ni de la psicología. En cambio, su aproximación se sitúa en el ámbito más amplio y difuso de la teoría-crítica y los estudios culturales. Desde allí, pretende descubrir los imaginarios sociales en torno a la niñez en objetos tales como el cine y la literatura.

Por otra parte, el título de este trabajo induce intencionadamente a un equívoco. La ambigüedad de la palabra queer -en inglés, a la vez extraño y homosexual- sugiere interpretar este libro desde una perspectiva de género $^{1}$. Esta lectura banaliza la propuesta central del texto y la reduce

1 La Revista de crítica cultural publica, en su $N^{\circ} 25$, un intercambio entre Bolívar Echeverría y Marta Lamas, en el que se discute el sentido de la palabra queer y el ingreso de este concepto al campo de los estudios de género de la academia hispanohablante. Al respecto, Bolívar Echeverría señala: "pienso que la palabra queer ('raro', chueco, sin cabida por atravesado, contrario de square, 'cuadrado', excesivamente normal) se debería entender el atributo distintivo de un comportamiento que sin ser 'de este mundo' se atreve a hacerse presente en él. [...] La queernes sería como el anuncio de una 'naturalidad' o 'normalidad' utópica o mesiánica para la vida humana; una forma de vida cotidiana basada en una experiencia puramente virtual o supuesta (siempre deseada, reclamada), la de la abundancia. [...] Y es ella también la que se reivindica y defiende en la actividad estética (y cultural) de los manieristas y barrocos a través precisamente de este regodeo en el artificio, que los vuelve chocantes para el gusto de la modernidad realista: extravagantes, bizarres, inconsistentes, vacíos" (56). 
(como la lectura de Salamone) a una defensa de la idea de que todo niño es homosexual. Esta interpretación no solo desplaza el texto de Kathryn Bond fuera de su espacio teórico más genuino, sino que elude las cuestiones más interesantes planteadas por el libro que tienen que ver con el lugar del niño y de la infancia en el imaginario de la modernidad, las perversiones que encierran dichos imaginarios y las metáforas al interior de las que los niños encuentran un escape a las constricciones impuestas a ellos por un mundo adulto del que son, a la vez, protegidos y excluidos. Para soslayar estos equívocos, este comentario comenzará refiriéndose a los problemas de traducción que The Queer Child propone a un lector hispanohablante.

\section{Problemas de traducción}

El término queer es algo más que una palabra

de difícil traducción en español además de ser precisamente eso: un término generado en una cultura diferente de la nuestra, el cual no tiene un equivalente que nos acerque de manera inmediata al sentido que en inglés evoca. - Marta Lamas ${ }^{2}$

No hay una forma adecuada de traducir la palabra queer al español. La palabra gay aparece como un sinónimo cercano pero, por cierto, esta es también un préstamo excéntrico a la lengua ${ }^{3}$. Por otra parte, en el mundo hispanohablante, gay se utiliza casi inequívocamente para significar "homosexual" y pierde el matiz benévolo y alegre que hace de esta una palabra tradicionalmente (aunque no actualmente) ambigua en su lengua original. Algo similar ocurre con la palabra queer. En nuestra lengua, el significado de esta palabra se sitúa en un espacio sin nombre, en algún punto entre "raro", "inadecuado", "excéntrico" y "torcido"; pero también "perverso", "homosexual", "marica" y "afeminado". De todas

2 Publicado en Debate Feminista 16 (1997), y citado en la Revista de crítica cultural 25 (2001).

3 También Bond Stockton entiende la palabra gay como sinónimo de homosexual en inglés. De esta manera, mientras queer es una palabra semánticamente laxa y ambigua, gay ha devenido un concepto estático. Como sinónimo de homosexual, gay se inscribe en el binomio heterosexualhomosexual, recto o incorrecto: "The authors also tell us that 'that's so gay' has 'become the mantra' of young 'school children' (one senses that this has been true for some time) and that 'eight year olds already know that «gay» equals «bad»'." (49). 
estas, las traducciones al sociolecto chilensis como "loca" o "mariquita" serían, tal vez, las menos insultantes. Estas palabras logran, al menos, retener algo de la ambigüedad del vocablo en su tradición original. Una mariquita es, después de todo, un pequeño bicho raro, delicado y hermosamente vestido -un Oscar Wilde entre los insectos, ataviado con una sedosa levita roja-.

En su libro, Kathryn Bond usa la palabra queer de manera amplia, e intencionadamente ambigua, para referirse a los niños que se encuentran, de alguna u otra forma, fuera de lugar: "If you scratch a child, you'll find a queer, in the sense of 'gay' or just plain strange" (Bond 1). La pregunta por la homosexualidad infantil aparece en este texto para relevar la pregunta más amplia sobre la posibilidad de conocer la infancia. Tal como lo explica la autora, cuando una persona puede preguntarse por su propia niñez e intentar comprender esa dimensión de su identidad, esa parte de sí se encuentra irremediablemente perdida. Su recuperación implica, necesariamente, la construcción de una narrativa y la re-significación de un grupo de imágenes fragmentarias y dispersas. Así, cuando una persona, en algún punto entre la adolescencia y la adultez, se re-conoce homosexual, descubre a su vez que fue un niño homosexual. De este modo, ser un niño gay nunca es un hecho presente, sino más bien la resignificación de un espacio temporal ya lejano y perdido.

A propósito, Kathryn Bond cita Queering Elementary Education como ejemplo de un grupo de publicaciones que pretenden educar en el respeto de la diferencia y abogan por la creación de ambientes escolares acogedores con la diversidad sexual. La autora subraya que, aun en este grupo de textos percibidos como progresistas, es posible encontrar bastantes sombras en torno a la idea de la homosexualidad en la infancia, "a fair amount of ghosting" (48). Estas sombras o fantasmas se advierten, por ejemplo, en la forma artificial en que estos textos soslayan hablar de niños "gay" a través de paráfrasis complicadas como "eventually emerging sexual selves" (49) o "children who develop into gay [...] people" (49). Parece entonces que en la infancia no se puede ser gay, aunque sí estar acompañado de esta suerte de premonición; un fantasma que anuncia que algún día se llegará a ser una persona homosexual. La identidad sexual en la infancia se puede construir solamente a través de este retorno nostálgico sobre las imágenes y recuerdos de la niñez. Esta mirada "hacia atrás" necesariamente desfigura los hechos y parece 
construir ficciones más que descubrir una verdad sobre el propio serniño. Solo que éste parece ser siempre el caso con la infancia. Por eso, más que una afirmación o una exploración de la forma en que se manifiesta la homosexualidad en la infancia, el texto levanta una pregunta en torno a la niñez y las ficciones adultas que le dan forma.

En la opinión pública, los niños se suponen inocentes, asexuados (o al menos sexualmente neutros), privados de la capacidad de decidir sobre sus acciones -incluso aquellas que les atañen más directamente, como su propio placer o dolor-. Bond agrega que en el concepto de niño, este se concibe blanco y de clase media. La idea de un niño gay desafía, por cierto, estas concepciones, pero también lo hacen todos los niños que disienten de este concepto elaborado por el adulto moderno y se sienten de alguna u otra forma fuera de lugar: "...this feeling of fearful self-disclosure may concern any child who feels out of sync with the children around her or feels repelled by the future being mapped for her" (52). Estos niños que se saben extraños, queer, deben encontrar formas laterales de crecer, "ways of growing which are not growing up" (Bond 11). Es decir, formas alternativas de vivir su infancia y que les permite imaginar un futuro distinto al imaginado y diseñado para ellos, imaginario en el que los niños normales e inocentes crecen para convertirse en adultos maduros, normales. Los niños queer crecen "sideways": hacia los márgenes de las normas de los adultos.

Nuevamente, traducir la expresión "to grow sideways" resulta complejo, y en esta se pierden muchas de las sutilezas imbricadas en su campo semántico. En inglés, la manera de referirse al crecimiento específicamente humano es "to grow-up", es decir, crecer hacia arriba, verticalmente, hasta alcanzar la estatura adulta. Esta forma de concebir el crecimiento encierra al menos dos ideas erradas o inexactas. La primera es que el crecimiento, y por lo tanto el desarrollo, se detiene al alcanzar la edad adulta. La segunda, es que hay una forma (co)rrecta de crecer: desde una infancia inocente y sexualmente suspendida hacia la adultez heterosexual. $\mathrm{Al}$ interior de estos estrechos límites creados por el mundo adulto, "The child who by reigning cultural definitions can't 'grow up' grows to the side of cultural ideals" (13). Esta es, ciertamente, la forma en que debe crecer el niño homosexual; en los márgenes, torcido hacia los límites externos del camino recto hacia la adultez heterosexual. Pero es también la forma en que casi todos los niños crecen; puesto que, en la 
modernidad, la infancia es un artificio por medio del cual los adultos han vuelto a los niños seres extraños y lejanos -queer-.

\section{El lugar del niño queer: imaginario, metáfora y nostalgia}

Las investigaciones de deMause y Ariès, mencionados anteriormente, discuten y disienten sobre el momento en que surge la infancia como etapa socialmente diferenciada y reconocida. Al respecto, deMause sugiere que la niñez siempre ha sido parte de la existencia humana, y que su estudio es crucial para comprender la historia social del hombre. Ariès, por su parte, sostiene que la infancia no existió como tal sino hasta los siglos XVII y XVIII, período en el que surgen clases medias acomodadas que podían permitirse prescindir del trabajo infantil. Puesto que el trabajo de Bond Stockton se sitúa en el siglo XX, estas discusiones le son ajenas. En cambio, en "el siglo de los niños" -momento histórico en que estos son reconocidos y protegidos, social y legalmente-, la autora descubre las muchas formas en que los niños subvierten las concepciones adultas sobre la infancia, a la vez que revela las perversiones y peligros que subyacen a estas.

Es así que, en la introducción al libro, la autora distingue cuatro formas en que los niños son enrarecidos por la normatividad adulta. Aparecen, así, el niño fantasmagóricamente gay, el homosexual adulto, el niño enrarecido (queered) por Freud, y el niño enrarecido por la inocencia o por el color ${ }^{4}$. De acuerdo a Bond, hacia finales del siglo XX, la literatura de auto-ayuda estadounidense comienza a evidenciar la necesidad social emergente de enfrentar el descubrimiento del niño homosexual. Los numerosos casos y testimonios contenidos en esos textos relatan las historias de jóvenes que, habiendo descubierto su homosexualidad durante la infancia, tuvieron que esconderla y encontrar maneras oblicuas de relacionarse con su identidad sexual. Para ellos -y para sus familias - el niño gay fue siempre un fantasma: una serie de indicios constantemente presentes, escondidos detrás del hijo que los padres a un tiempo veían e imaginaban. Así, la revelación de la homosexualidad del hijo es enfrentada por los padres como la muerte de un ser querido;

4 En el texto original: the ghostly gay child, the grown homosexual, the child queered by Freud y the child queered by innocence or queered by color. (Las cursivas son propias). 
más aun: "The previously beloved son or daughter suddenly seems to disappear from life and is replaced by a sinister version of the same person" (19-20). Para el hijo, en cambio, se trata de un doble nacimiento; de un nacimiento invertido -a backward birth-, puesto que solo entonces puede construir la historia de su niñez como un continuo hasta la vida adulta.

Un ejemplo de estas apariciones fantasmagóricas del niño homosexual es explorado por la autora a través de la película The hanging garden (Thom Fitzgerald, 1997). En ésta, un hombre homosexual exitoso, con una vida económica y emocionalmente estable, vuelve a la casa de sus padres con motivo del matrimonio de su hermana. Allí se reencuentra con los lugares y memorias conflictuados de su infancia y adolescencia: "As the film unfolds, viewers see his fat-teen self not only as a character in flashback (eating, being lonely, being hit by his father, having sex with a boy) but also as the film's primary ghost -since an image of him hanging dead above the garden keeps appearing to the viewer [...] and to several other characters" (20).

Por supuesto, el fantasma no es solo esta imagen del yo-adolescente del protagonista que aparece ahora para asediarlo en su retorno a casa. Durante toda su infancia, su queernes estuvo presente como un fantasma, disfrazada de niño obeso. "We see this ghost child in the kitchen, his hand in the cookie jar as the gay man tells his mother: 'Skinny was the only thing you could never make me be'" (20). De esta forma, ser gordo fue un signo permanente de la rareza del protagonista durante su niñez. Pero también fue una forma de ser queer a pesar de las ideas adultas sobre lo que debería ser un niño.

Como la literatura y los fantasmas, las relaciones entre niños y adultos son un entramado de imaginaciones: la imaginación del adulto de lo que fue su niñez, la imaginación del niño acerca de lo que será cuando crezca, la imaginación del niño acerca de sí mismo y de la idea que tienen de él los adultos. A propósito, la autora establece un paralelo entre los mecanismos que el niño queer utiliza para relacionarse con su propia no-normatividad y la metáfora, que es la forma en que el lenguaje se imagina otro: "Perhaps this circumstance of the gay child leads the child to metaphor, which itself is a sideways accretion: the act of imagining oneself as something else" (15). Los crecimientos laterales son, 
así, espacios para la metáfora, formas de ser niño que soslayan los límites impuestos por el mundo adulto. En suma, formas de ser otros. De este modo, los crecimientos laterales -metafóricos- establecen un paralelo entre la imaginación de la infancia y la literatura que sugiere perseguir las ficciones al interior de las que habitamos. Bond propone buscarlas, precisamente, en la ficción.

A lo largo del libro la autora identifica tres ámbitos de crecimiento lateral: "sideways relations, motions and futures" (52). En la literatura y el cine, Bond busca estas formas laterales -metafóricas- de explorar los sueños y deseos durante la infancia, y a pesar de ser niño: "[I seek to theorize] a new view of metaphor, one grasped not solely in terms of translation (the girl as dog) but also in terms of transport and time (a girl who is moving through an interval of time). This makes metaphor a moving suspension" (53).

En la novela The Pupil, de Henry James, se muestra cómo un jovencito manipula el deseo de su tutor y logra, de esta forma, tanto librarse de sus padres como decidir sobre su propia educación. Entre the pupil y su tutor se establece un tipo de relación lateral, a sideways relation, que les permite a ambos explorar en la relación hombre-niño que les está permitida -la del alumno y su tutor- los sueños y deseos sexuales que les están prohibidos. Por otra parte, Kathryn Bond descubre cómo los personajes encuentran en la manipulación y el dolor, una forma de placer en la dilación, "the promise of a love growing sideways" (53).

Más adelante, el libro examina las relaciones laterales entre las niñas y los perros. "The animal in novel by Virginia Woolf, Djuana Barnes, and Radcliffe Hall is a different kind of peer in the game of delay. It is, at turns, a witness, confidante, rebel, protector, and pretend lover" (53). Bond explora cómo los perros aparecen como canales de los deseos y afectos de las niñas, pero también, cómo estos animales constituyen metáforas de las niñas mismas. Estos son paralelos de su crecimiento lateral, puesto que su crecimiento también ocurre fuera de los tiempos de desarrollo humano. Por otra parte, la autora explica que los perros, junto a los vehículos motorizados, constituyen metáforas de los motivos infantiles. En Lolita, de Nabokov, por ejemplo, son un perro y un automóvil los que causan la muerte de Charlotte, uniendo de manera irremediable su hija Dolly a Humbert Humbert, "a dog beside a girl [...] might express the 
feelings of the girl by its side [...]. [L]ocomotions, not just postures or facial expressions, of human beings -on their legs or in their vehiclesmight indicate specific emotions, even motives" (54).

Esto último conduce la discusión al problema del motivo infantil. A la luz de los sistemas legales del siglo XX, los niños no solo son incapaces de tomar decisiones respecto a su vida sexual, sino que también son inimputables desde el punto de vista penal. Esto último problematiza el hecho mismo de que los niños puedan tener intenciones criminales o concebir motivos para cometer un delito. Desde las categorías de análisis propuestas por Bond, el motivo infantil puede ser concebido -de manera similar al desarrollo de una identidad sexual en la infancia- como un crecimiento oblicuo. Al momento de perpetuarse la acción criminal, surge la búsqueda de la serie de vivencias, emociones y traumas que configuran los motivos e intenciones del niño. La autora descubre estos "motive[s] growing sideways" en In Cold Blood, de Truman Capote (1965), Heavenly Creatures, de Peter Jackson (1994), y abre nuevas preguntas sobre los problemas jurídicos del motivo infantil a partir de la película Elephant, de Gus van Sant (2003).

Hacia el final del libro, el modelo de análisis levantado por Bond prueba ir más allá de un estudio de lo queer en la infancia. La autora analiza la relación de la población blanca estadounidense con sus ideas sobre el mundo negro, por una parte, y con la forma en que se relaciona con este mundo en los hechos, por otra. Así, los niños soldados de la película Blood Diamond (Edward Zwick, 2006), exponen cómo los niños de África desafían la idea del niño inocente (blanco, de clase media y protegido del daño y el peligro). La realidad de estos niños asesinos problematiza dramáticamente el estatus jurídico de los niños en América; ás aún, puesto que la africana es una otredad siempre presente en Estados Unidos. El encuentro con esta otredad se pone a prueba en Guess Who's Comming to Dinner (1967). En este filme de Stanley Kramer, un matrimonio liberal conoce al prometido de su hija: un joven médico afroamericano. Si el concepto de un crecimiento oblicuo da cuenta de los problemas que genera el concepto de infancia al interior de la sociedad en su orden jurídico, el nacimiento invertido que puede describir la experiencia de re-conocimiento del niño homosexual descubre, también, el nacimiento invertido de la sociedad blanca-liberal al encuentro con sus propias ideologías. 
Por último, de acuerdo al documental Hoop dreams (Steve James, 1994) y a la película Charlie and the Chocolate Factory (Tim Burton, 2005), Bond pone de relieve el permanente contacto de los niños con la cultura de consumo y las maneras en que estos se hacen vulnerables a causa de su fascinación con los objetos de deseo que la propaganda pone a la vez tan cerca y tan lejos de su alcance. Hoop dreams muestra la vida de tres jóvenes afroamericanos de sectores socioeconómicos vulnerables en su intento por convertirse en jugadores profesionales de baloncesto. A medida que el filme se desarrolla, el espectador comprende que el futuro prometido -los sueños auspiciados por Nike- se dirigen al fracaso. Charlie and the Chocolate Factory, en cambio, dibuja metáforas más dulces de la crueldad con que el mercado seduce a los niños: "As if replying to the need to fill shoes and pass on dreams, even fulfill them for white children, Charlie and the chocolate Factory [...] imagines one can manufacture dreams" (238). Que los niños sueñan con objetos y caramelos extiende la pregunta sobre el poder de manipulación que el mundo del dinero ejerce sobre los niños. La película de Tim Burton (y la obra de Roald Dahl) sugiere que este poder puede empujarlos a su auto-destrucción, o a veces, (peligrosamente) cerca de la compañía de los fabricantes de sueños.

\section{Tiempo, dilación, inocencia}

Si la nostalgia se vuelve en retrospectiva sobre el espacio perdido de la infancia, esta última se crea en virtud de una postergación de la adultez. Esta dilación del crecimiento es creada por los adultos para el desarrollo "adecuado" de los niños. En palabras de la autora, "Delay is seen as a friend to the child. Delay is said to be a feature of its growth: children grow by delaying their approach to the realms of sexuality, labor and harm ${ }^{5 \prime}(62)$. Esta dilación consiste y puede solo consistir en un retraso de las acciones y decisiones del niño respecto a estos ámbitos, puesto que el contacto de los niños con estas realidades es, a pesar de todo, ineludible. Postergar la actividad sexual parece ser una forma alternativa de relacionarse con el sexo; es "an active arrest" (63), en el cual solo tienen lugar diversiones alternativas al sexo que, por eso mismo, son intensamente sexuales. 
Más aun, aunque desde Freud es insostenible proponer que los niños sean seres a-sexuados, muchas veces pareciera que sí se los concibe como seres neutros o al menos, sexualmente suspendidos. De modo similar, aunque desde inicios del siglo $\mathrm{XX}$ los niños han salido del mercado laboral, no por ello han dejado de estar en contacto con el dinero. De hecho, en este período, en lugar de un salario, los niños reciben mesadas y regalos que los convierten en activos consumidores de caramelos y juguetes. Lolita, por ejemplo, es constantemente seducida por anuncios comerciales: "Advertisements -and the objects they lure consumers into (Quilty, as it happens, is one of such objects) -are far more powerful in seducing Dolly than Humbert ever imagines he could be. And, of course, its money that makes Lolita stay with Humbert Humbert ('she had absolutely nowhere to go')" (225).

A medida que la novela avanza, cada vez con mayor frecuencia Lolita cambia favores sexuales a Humbert por dinero. De esta forma, la dilación modela las formas en que los niños se relacionan con y transgreden los espacios del sexo y el dinero; construye unos márgenes al interior de los cuales establecen relaciones oblicuas con estos espacios de la vida social de los cuales se encuentran, supuestamente, exentos.

Curiosamente, la dilación o postergación ha sido una de las formas tradicionales de entender la homosexualidad adulta. Freud mismo describe la homosexualidad no como una perversión o enfermedad, sino como una detención del desarrollo; una suerte de permanencia en el período infantil. Los adultos homosexuales, según las anotaciones de Freud, no se sienten plenamente hombres adultos, sino más bien niños o adolescentes. A propósito, Bond desliza una reflexión acerca de las posibilidades de complicidad que pueden emerger entre los niños queer y los adultos homosexuales, ambos detenidos en un crecimiento oblicuo. La relación problemática entre el niño y su tutor en The Pupil de Henry James sugiere, ciertamente, este tipo de vínculo. De algún modo, también lo hace el curioso encuentro entre Charlie y Willie Wonka, al menos en la forma en que Tim Burton se apropia del relato de Roald Dahl.

Este último es un pensamiento perturbador y molesto porque transgrede la concepción más general y básica sobre la infancia: la inocencia. Ahora, si Charlie and the Chocolate Factory y The Pupil son algunos ejemplos de cómo la ficción ilustra estas relaciones oblicuas, Kathryn Bond se hace cargo de poner estos ejemplos en perspectiva por 
medio de casos de no-ficción como el movimiento NAMBLA. Este es un movimiento estadounidense que defiende las relaciones amorosas entre niños y adultos. Aunque la organización no promueve las relaciones sexuales entre adultos y menores, sí cuestiona el hecho de que estas deban resultar necesariamente dañinas para el niño, y por sobre todo, cuestiona la tajante negativa a que los niños puedan consentir en sostener relaciones sexuales con otros niños o con personas mayores. Más allá del repudio que inspira al sentido común moderno la idea de que las relaciones entre pedófilos y niños homosexuales pueden ser amistosas (aun benignas), el libro de Bond invita a contemplar desprejuiciadamente ciertas realidades que -no obstante el repudio- tienen lugar en la sociedad. Su trabajo arroja luz sobre los parámetros de "normal" y "a-normal", "straight" y "queer" al interior de los que elegimos vivir. El objetivo de todo esto no es, precisamente, enjuiciar dichos parámetros, pero explicitar sus límites y señalar sus peligros.

Protegidos y excluidos de la relación directa con el mundo del dinero y el sexo, a la vez privados de agencia, pero en permanente contacto con estos ámbitos, los niños se vuelven extraños y distantes a causa de su identificación con la inocencia: "What do children queered by innocence share? They all share estrangement from what they approach: the adulthood against which they must be defined. This is why 'innocent' children are strange. They are seen as normative but also not like us, at the same time" (31).

Ser la encarnación de esta idea de inocencia hace a los niños dignos de protección. Paradójicamente, también acentúa su vulnerabilidad y los vuelve peligrosos: "Innocence, that is, works its own violence on adults and children" (12). Es la misma lejanía y rareza de los niños inocentes la que los vuelve excitantes para los adultos. De esta forma, se crea para los niños una esfera de protección que los vuelve objetos deseables. Son una amenaza para sí mismos y también para los adultos que se sienten (culposamente) excitados por su fragilidad y su belleza.

La peligrosidad de los niños -y su inocencia- es acentuada por su inimputabilidad penal. En este sentido, es que "[t]he child is [...] defined as a kind of legal strangeness. It is a body said to need more protections than freedoms. And it is a creature that cannot consent to its sexual pleasure, or divorce its parents, or design its education -at least not by law" (16). El libro de Bond Stockton recorre la literatura y el cine en 
busca de niños que subvierten y desafían esta concepción adulta de la inocencia. Si confiamos en la propuesta de Ariès y deMause respecto a la importancia de comprender la infancia para escribir una historia del hombre, entonces el libro de Bond es una pieza importante para construir una historia del siglo $\mathrm{XX}$ a través de sus manifestaciones culturales. Los ejemplos literarios y fílmicos analizados por la historia constituyen, en efecto, una evidencia de la cultura contemporánea en torno a los niños. Aún más, las categorías que la autora levanta en este trabajo -desde un análisis de lo queer o de la homosexualidad en la infancia-pueden ser extrapoladas al análisis de otras formas de no-normatividad: la diferencia de color, o de los sujetos que son excluidos de los márgenes de la vida política y jurídica. Lo que lo queer -en sentido amplio- permite poner en evidencia es, entonces, cómo se construyen los espacios normativos de lo correcto y lo incorrecto, lo aceptable y lo inaceptable, lo que puede ser y lo que debe ser temporalmente suspendido (delayed).

Por supuesto, Kathryn Bond piensa la infancia -y las categorías metafóricas que se desprenden de pensar su queernes- desde (y para) el contexto de Estados Unidos. Por lo mismo, sus ejemplos provienen de la cultura norteamericana y hablan del niño queer "gringo". En efecto, tal como señala Marta Lamas "[e]l término queer es algo más que una palabra de difícil traducción en español" (Lamas, 2001, 56). Con todo, acaso es posible desplazar las categorías de crecimientos oblicuos, nacimientos invertidos y otredad queer de la inocencia infantil hacia las obras de la literatura latinoamericana del siglo XX. Inmediatamente aparecen ejemplos de niños en nuestra literatura que desafían la idea americana de niño -esa que aparece en los comerciales de Simond's y de papel higiénico Elite-. Entre ellos, el Silvio Astier de Roberto Artl o el niño proletario de Osvaldo Lamborghini, que cuestionan la inocencia a-priori en la que los sistemas penales sitúan a los niños. Otros como la marquesita de José Donoso o incluso Hans Pozo desestabilizan las pre-concepciones adultas sobre las relaciones entre los niños y el sexo. Con todo, hablar de los niños en la literatura de Latinoamérica implica más de algún ejercicio de traducción. Requeriría definir la idea de niño desde las características específicas de este espacio. Acá es posible que lo queer en la infancia se manifieste en crecimientos oblicuos en relación al sexo, a través de caramelos y zapatillas Nike, pero también el futbol, el reggaetón y la pasta base. 


\section{Referencias Bibliográficas}

Ariès, Philippe. Centuries of Childhood: a social history of family life. Trad. Robert Baldick. Nueva York: Vintage, 1962. Impreso.

Blood diamond. Dirección por Edward Zwick, reparto Leonardo DiCaprio, Jennifer Connelly y Djimon Hounsou. Productor, Edward Zwick (2006).

Bond Stockton, Kathryn. The Queer Child or Growing Sideways in the Twentieth Century. Durham: Duke University Press, 2009. Impreso.

Charlie and the Chocolate Factory. Dirección por Tim Burton, reparto Johnny Depp y Freddie Highmore con David Kelly, Helena Bonham Carter, Noah Taylor, Missi Pyle, James Fox, Deep Roy, Christopher Lee. Productora, Village Roadshow Pictures, The Zanuck Company, Plan B Entertainment. Distribuidora Warner Bross Pictures (2005).

DeMause, Lloyd. The history of Childhood. Nueva York: Harper Torchbooks, 1975. Impreso.

Echevarría, Bolívar. «Queer, manierista, bizarre, barroco.» Revista de crítica cultural 25 (2001): 56-58. Impreso.

Elephant. Dirección por Gus Van Sant, reparto Alex Frost, Eric Deulen, John Robinson, Elías McConnell, Jordan Taylor, Carrie Finklea, Nicole George, Alicia Miles, Timothy Bottoms, y Matt Malloy. Productores, Diane Keaton, Dany Wolf y JT LeRoy (2003).

Guess Who's Coming to Dinner. Dirección por Stanley Kramer, reparto Spencer Tracy, Katharine Hepburn, Sidney Poitier y Katharine Houghton. Productor, Stanley Kramer (1967).

Freeman, Elizabeth. «Review: STOCKTON, KATHRYN BOND. The Queer Child: Growing Sideways in the Twentieth Century.» Studies in the Novel 43.1 (2009): 128130. Electrónico (URL): http://www.jstor.org/ stable/ 41203510 . 
Hoop Dreams. Dirección por Steve James. Productores, Steve James, Peter Gilbert, Frederick Marx, con Kartemquin Films. Distribuida por Fine Line Features (1994).

James, Henry. The Pupil, ed. David Price. Boston, Le Roy Philips (1916). [Reproducido por Gutenberg Project]. Electrónico (URL): http:/ / www.gutenberg.org/ files / 1032/1032-h/1032-h.htm

Romesburg, Don. «Review of Stockton, Kathryn Bond, The Qeer Child, or Growing Sideways in the Twentieth.» H-Childhood, H-Net Reviews (2010). Electrónico (URL): http:/ / www.h-net.org/ reviews / showrev.php?id=29492.

Salamone, Frank A. «Review: Kathryn Bond Stockton (2009) The Queer Child, or Growing Sideways in the Twentieth Century.» Childhood 19.2 (2012): 282-283. Electrónico. 